\title{
EVIDENCE FOR COLLATERAL PROJECTIONS BY NEURONS IN AMMON'S HORN, THE DENTATE GYRUS, AND THE SUBICULUM: A MULTIPLE RETROGRADE LABELING STUDY IN THE RAT ${ }^{1}$
}

\author{
L. W. SWANSON, P. E. SAWCHENKO, AND W. M. COWAN
}

The Salk Institute for Biological Studies, La Jolla, California 92037

\begin{abstract}
Although it has been recognized for some years that each cytoarchitectonic field of Ammon's horn and the subiculum gives rise to a specific pattern of cortical and subcortical projections, it has not been clear whether these various projections arise from different populations of neurons within each field or whether they arise as collaterals from an essentially homogeneous population of cells. We have examined this problem, and the related issue of the origin of the commissural and ipsilateral associational projections of the dentate gyrus, by injecting retrogradely transported fluorescent dyes into two or more of the relevant projection fields in adult rats and subsequently examining the brains for doubly or triply labeled neurons.

It is clear from these experiments that at least two of the known efferent projections of field $\mathrm{CA}_{1}$ (to the septum and to the entorhinal cortex) arise from the same pyramidal neurons and also that the commissural, ipsilateral associational, septal, and subicular projections of the other major field of Ammon's horn-field $\mathrm{CA}_{3}$-similarly are due to collaterals. Double-labeling experiments also indicate that at least $80 \%$ of the cells in the deep hilar region of the dentate gyrus give rise to both an ipsilateral (associational) and a crossed (or commissural) projection to the dentate granule cells. In contrast, the projection neurons in the dorsal part of the subiculum form a quite heterogeneous population; cells that project to both the septum and the entorhinal area are intermingled with others that project to one or the other area but not to both. The cortical and cortico-subcortical connections of the hippocampal formation thus appear to be quite different from those of the neocortex, and the existence of such an extensive system of collateral projections clearly has important consequences for studies of the development of the hippocampus and of its response to selective deafferentation.
\end{abstract}

It has been known for some years that each of the fields of Ammon's horn (or hippocampus proper) projects to a number of areas within the forebrain. For example, each division within the field designated " $\mathrm{CA}_{3}$ " by Lorente de Nó (1934) has been found to project to the corresponding hippocampal field on the opposite side, to Lorente de Nó's field $\mathrm{CA}_{1}$ on both sides, and to the subicular complex and the lateral septal region of both sides. Similarly, field $\mathrm{CA}_{1}$ has been shown to project both rostrally to the lateral septal nucleus (on the same side) and caudally to the subiculum and the entorhinal area (see Swanson and Cowan, 1977 for a summary of the experimental evidence on which these conclusions are

This work was supported in part by the Clayton Foundation for Research and in part by Grants NS-13267 and NS-16980 from the National Institutes of Health and Grant DA-00259 from the Alcohol, Drug Abuse, and Mental Health Administration. W. M. Cowan is a Senior Investigator of the Clayton Foundation. We would like to thank Mr. Laurence Schmued for his help in preparing some of the experimental material and Ms. Pat Thomas for typing the manuscript. based). More recently, it has been found that the subiculum itself has both cortical and subcortical projections to the entorhinal area and to the septum and hypothalamus, respectively (Swanson and Cowan, 1975; Meibach and Siegel, 1975; Chronister et al., 1975). Now, there is also evidence that certain cells in the deep hilar zone of the dentate gyrus give rise to an ipsilateral (associational) and a crossed (or commissural) projection to the dentate granule cells (Zimmer, 1971; Gottlieb and Cowan, 1972, 1973; Hjorth-Simonsen and Laurberg, 1977; Amaral, 1978; West et al., 1979). However, it is not known whether these various projections each arise from discrete subpopulations of neurons within the relevant cytoarchitectonic fields or whether they arise entirely, or in part, as collateral projections from essentially homogeneous populations of neurons.

In Golgi preparations, one often can see collateral branches arising from the axons of hippocampal pyramidal cells (Ramón y Cajal, 1911; Lorente de Nó, 1934) and from certain cells in the hilar region of the dentate gyrus (Amaral, 1978), but with the exception of the myelinated 
collaterals that arise from the axons of the large pyramidal cells of field $\mathrm{CA}_{3}$, which can be traced to field $\mathrm{CA}_{1}$ (the so-called "Schaffer collaterals"), it has been impossible to determine the distribution of all but the shortest of these collaterals.

When the technique that is based on the retrograde transport of horseradish peroxidase (HRP) was first introduced for use in the central nervous system (LaVail and LaVail, 1972), we attempted to address this issue by placing injections of the enzyme into each of the major projection fields of the hippocampal formation and subsequently estimating the numbers of retrogradely labeled neurons. If all, or even a majority, of the neurons in a particular field could be labeled after a single injection of HRP, this would have been strong presumptive evidence that at least some of the projections of that field must arise as collaterals. Unfortunately, we were never able to label more than $30 \%$ to $40 \%$ of the relevant pyramidal cells after injections into the hippocampus of the opposite side, into the septal region of both sides, or into the entorhinal area. As this was true even when we used the more sensitive method suggested by Mesulam (1976), the issue was left unresolved because it was unclear whether the limited number of neurons that were labeled was an accurate reflection of an inherent heterogeneity within the pyramidal cell population or whether it was due to some limitation in the HRP method (Jones and Leavitt, 1974; Jones and Hartman, 1978). The uncertain sensitivity of the HRP method also deterred us from attempting to address the problem with the other methods that were available then which involved the use of HRP either alone (De Olmos and Heimer, 1977) or in conjunction with a second retrograde tracer such as ${ }^{3} \mathrm{H}$-labeled HRP or ${ }^{3} \mathrm{H}$-labeled bovine serum albumen (Geisert, 1976; Steward et al., 1977), although these methods had been used successfully to identify collateral projections in the hippocampal formation and elsewhere.

In the past 2 or 3 years, Kuypers and his colleagues have introduced a number of new, retrogradely transported markers into neuroanatomy (Kuypers et al., 1977, 1979; Bentivoglio et al., 1979, 1980b). Most of these are fluorescent dyes that are taken up by axons and retrogradely transported, at different rates, to the cell somata where they appear to bind selectively to various nuclear or cytoplasmic components. As they have different emission spectra, they can be used to label neurons that have collateral projections by simply injecting two or more dyes into the relevant projection areas and, after suitable post-injection survival periods, examining the labeled cells in a fluorescence microscope. The identification of doubly (or multiply) labeled cells provides direct evidence that the neurons in question have two or more collateral projections (Van der Kooy et al., 1978). Conversely, if all of the neurons in a particular region are labeled retrogradely after a single injection, then it follows that any other projection from that population of neurons must arise by collateral branching of the cells' axons.

In an earlier study (Swanson et al., 1980), we used both of these approaches to analyze the origins of the various efferent projections of the major field of the regio inferior of Ammon's horn. The findings in both cases were consistent in showing that, from the point of view of their projections, the pyramidal cells of field $\mathrm{CA}_{3}$ form an essentially homogeneous population because virtually all of the cells in any given sector of the field could be labeled retrogradely by single injections in any of several of the projection areas, and because most of the cells could be doubly or triply labeled after appropriately placed injections of two or three different dyes. We now have extended this analysis to include field $\mathrm{CA}_{1}$ of Ammon's horn, as well as certain of the efferent projections of the subiculum, and using the same methods, we have determined the cells of origin of the commissural and associational afferents to the dentate gyrus.

\section{Materials and Methods}

A total of 42 adult albino rats was used in these experiments. In each case, one or more of the following dyes was injected stereotaxically into the various projection fields of the hippocampal formation: bisbenzimide ( $2 \%$ aqueous solution), Evans blue (10\% aqueous suspension), true blue ( $2 \%$ aqueous suspension), and nuclear yellow ( 1 to $5 \%$ aqueous suspension). For the injections, the animals were anesthetized with chloral hydrate $(1 \mathrm{ml}$ of a $3.5 \%$ solution/ $100 \mathrm{gm}$ of body weight) and mounted in a Kopf stereotaxic apparatus to which a $1-\mu \mathrm{l}$ Hamilton syringe was attached. Each injection consisted of 20 to $50 \mathrm{nl}$ delivered over a $15-\mathrm{min}$ period, after which, the needle was withdrawn and the skin was sutured. The animals were allowed to survive for different post-injection intervals in order to provide optimal retrograde labeling of the cells while, at the same time, minimizing leakage of the dye from retrogradely labeled neurons (Bentivoglio et al., 1980a; Swanson and Kuypers, 1980). The optimum survival time for each dye and each pathway was determined empirically in an initial group of control experiments. After the required survival period, the animals were perfused through the heart for 3 to 5 min with isotonic saline followed by $10 \%$ formalin in 0.1 M phosphate buffer at $\mathrm{pH} 7.4$ for $20 \mathrm{~min}$. The brains then were removed and cut into blocks which were placed in cold $\left(4^{\circ} \mathrm{C}\right)$ fixative containing $15 \%$ sucrose (Sawchenko and Swanson, 1981). A list of the successful experiments with details of the dyes used, the injection sites, and the survival periods is given in Table I.

The percentage of retrogradely labeled neurons in a particular field was estimated by counting the numbers of fluorescently labeled cells with distinct nucleoli and comparing this to the numbers of neurons that could be seen in the same area in an adjacent, Nissl-stained section. The localization of the retrogradely labeled cells was determined similarly with the aid of adjacent Nisslstained sections or, in some cases, by counterstaining the fluorescent sections with a dilute solution of acridine orange which provides the equivalent of a "fluorescent Nissl-stain" when viewed with Leitz filter system $I_{2}$ but is barely visible when viewed with filter system $A$ that is used for true blue, bisbenzimide, and nuclear yellow (L. Schmued, L. W. Swanson, and P. E. Sawchenko, manuscript in preparation).

\section{Results}

Two factors entered into the design and analysis of these experiments. The first concerns the possible involvement of preterminal fibers passing through, or close 
TABLE I

A summary of critical experiments with tracer dye injections in the hippocampal formation and septal region Only successful experiments with appropriate survival times and injection sites are indicated.

\begin{tabular}{|c|c|c|c|c|c|c|c|}
\hline $\begin{array}{c}\text { Injection } \\
\text { Site }^{a}\end{array}$ & $\begin{array}{c}\text { No. of } \\
\text { Animals } \\
\end{array}$ & Dye & $\begin{array}{c}\text { Survival } \\
\text { Time }\end{array}$ & $\begin{array}{c}\text { Injection } \\
\text { Site }^{a}\end{array}$ & $\begin{array}{c}\text { No. of } \\
\text { Animals }\end{array}$ & Dye & $\begin{array}{c}\text { Survival } \\
\text { Time }\end{array}$ \\
\hline & & & days & & & & days \\
\hline \multicolumn{4}{|c|}{ Retrograde Labeling in Hilar Region } & \multicolumn{4}{|c|}{ Retrograde Labeling in Field $C A_{3}$} \\
\hline One injection & & & & One injection & & & \\
\hline DG (L) & 2 & TB & 6 & HIP (L) & 1 & TB & 4 \\
\hline DG (L) & 1 & BB & 1 & HIP (L) & 1 & EB & 5 \\
\hline \multirow[t]{2}{*}{ DG (L) } & 2 & NY & 2 & HIP (L) & 1 & BB & 1 \\
\hline & \multirow[b]{3}{*}{2} & \multirow{3}{*}{$\begin{array}{l}\mathrm{TB} \\
\mathrm{NY}\end{array}$} & \multirow[b]{3}{*}{4} & SEPT (L) & 1 & $\mathrm{BB}$ & 1 \\
\hline Two injections & & & & $\mathrm{CA}_{1}$ & \multirow[t]{2}{*}{2} & \multirow[t]{2}{*}{ TB } & \multirow[t]{2}{*}{$3-5$} \\
\hline $\begin{array}{l}\text { DG (L) } \\
\text { DG (R) }\end{array}$ & & & & Two injections & & & \\
\hline DG (L) & \multirow[b]{2}{*}{2} & \multirow{2}{*}{$\begin{array}{l}\text { TB } \\
\text { BB }\end{array}$} & \multirow{2}{*}{$\begin{array}{l}4-6 \\
1\end{array}$} & HIP (L) & \multirow{2}{*}{3} & \multirow{2}{*}{$\begin{array}{l}\text { TB } \\
\text { BB }\end{array}$} & \multirow{2}{*}{$\begin{array}{l}4 \\
1\end{array}$} \\
\hline DG (R) & & & & HIP (R) & & & \\
\hline \multicolumn{3}{|c|}{ Retrograde Labeling in Field $C A_{1}$} & & $\begin{array}{l}\text { SEPT (L) } \\
\text { SEPT (R) }\end{array}$ & 2 & $\begin{array}{l}\text { TB } \\
\text { BB }\end{array}$ & $\begin{array}{l}4 \\
1\end{array}$ \\
\hline \multicolumn{4}{|c|}{ One injection } & $\mathrm{HIP}(\mathrm{L})$ & \multirow{4}{*}{1} & \multirow{4}{*}{$\begin{array}{l}\text { EB } \\
\text { TB }\end{array}$} & \multirow{4}{*}{$\begin{array}{l}4 \\
5\end{array}$} \\
\hline SEPT (R) & 1 & TB & 6 & SEPT (L) & & & \\
\hline ENT (R) & 1 & TB & 6 & & & & \\
\hline SUB (R) & \multirow[t]{3}{*}{1} & \multirow[t]{3}{*}{$\mathrm{TB}$} & \multirow[t]{2}{*}{3} & Three injections & & & \\
\hline Two iniections & & & & HIP (L) & & EB & 4 \\
\hline Two injections & & & & $\mathrm{HIP}(\mathbf{R})$ & 2 & BB & 1 \\
\hline SEPT (R) & \multirow[t]{2}{*}{1} & \multirow{2}{*}{$\begin{array}{l}\text { BB } \\
\text { TB }\end{array}$} & 1 & SEPT (L) & & TB & 4 \\
\hline SUB (R) & & & 5 & $\mathrm{HIP}(\mathrm{L})$ & & $\mathrm{TB}$ & 5 \\
\hline SEPT (R) & 1 & NY & 3 & HIP (R) & 1 & EB & 5 \\
\hline SUB (R) & 1 & TB & 3 & SEPT (R) & & BB & 1 \\
\hline SEPT (R) & 2 & BB & 1 & HIP (L) & & EB & 5 \\
\hline ENT (R) & 2 & TB & 5 & HIP (R) & 4 & TB & 5 \\
\hline SFPT (R) & & NY & 6 & SEPT (R) & & $\mathrm{BB}$ & 1 \\
\hline ENT (R) & 1 & TB & 6 & $\mathrm{HIP}(\mathrm{R})$ & & $\mathrm{EB}$ & 5 \\
\hline & & & & SEPT (R) & 4 & $\mathrm{BB}$ & 1 \\
\hline Retr & abeling $i$ & culum & & SEPT (L) & & TB & 5 \\
\hline Two injections & & & & & & & \\
\hline SEPT (R) & & BB & 1 & & & & \\
\hline ENT (R) & 2 & $\mathrm{~TB}$ & 5 & & & & \\
\hline SEPT (R) & & NY & 6 & & & & \\
\hline $\operatorname{ENT}(R)$ & 1 & TB & 6 & & & & \\
\hline
\end{tabular}

a The abbreviations used are: BB, bisbenzimide; DG, dentate gyrus and adjacent parts of Ammon's horn; EB, Evans blue; ENT, entorhinal area; HIP, hippocampus (Ammon's horn); L, left side of brain; NY, nuclear yellow; R, right side of brain; SEPT, septal region; SUB, subiculum; $\mathrm{TB}$, true blue.

to, the site of the dye injections. We have shown elsewhere (Sawchenko and Swanson, 1981) that both damaged and undamaged fibers can take up and retrogradely transport true blue and bisbenzimide. For this reason, when attempting to label intrahippocampal association fibers, we were careful to place the injections near the middle of the longitudinal (or septotemporal) extent of the hippocampus or dentate gyrus and to consider only those retrogradely labeled neurons that were located rostral to the injection site (Fig. 1). Because of the fiberof-passage problem, cells caudal to the injection site could be labeled either because they gave rise to ascending associational fibers or to commissural fibers that were destined for the opposite side by way of the ventral hippocampal commissure.

The second factor concerns the topographic arrangement of the various cortical and subcortical projections of the hippocampal formation. Autoradiographic studies have established that all of the hippocampal afferents are organized topographically, especially within the septotemporal dimension of each field. For example, the pyramidal cells in the septal pole of field $\mathrm{CA}_{3}$ have been found to project bilaterally to the dorsalmost part of the lateral septal nucleus and to the rostral (or septal) part of field $\mathrm{CA}_{1}$ on both sides of the brain, whereas cells in the temporal (or caudal) part of $\mathrm{CA}_{3}$ project to more ventral parts of the lateral septal nuclei and to the temporal parts of field $\mathrm{CA}_{1}$ (again, on both sides). Thus, if one is to label the cells with two or more dyes, it is important that the injection sites be chosen carefully and the injections be placed accurately. Because of the inherent inaccuracies in stereotaxic work (especially when multiple injections are made in the same brain) and because, in some cases (for example, the associational projections), the labeled cells may lie close to the injection site, multiple labeling of cells is found often over relatively small regions of the relevant hippocampal field. Furthermore, unless the survival time is optimal for each 

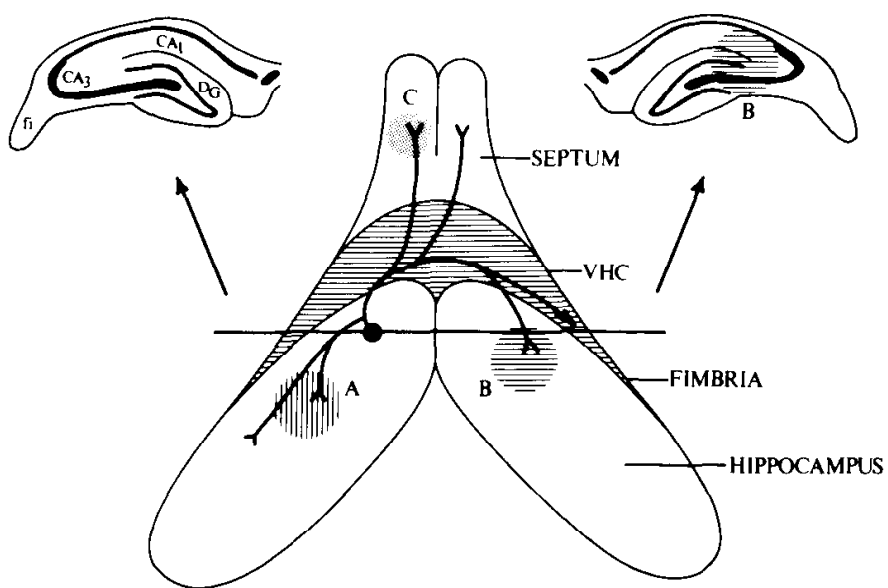

Figure 1. A schematic diagram of the principal efferent projections of a neuron in the regio inferior superimposed upon an outline of the rat hippocampus and septum as viewed from above. This figure illustrates the experimental approach used in all of the experiments in order to avoid labeling of fibers-ofpassage. For example, injections into the middle third of the ipsilateral hippocampus $(A)$ label cells that give rise to descending associational fibers, injections in the contralateral hippocampus $(B)$ label cells that contribute to the commissural projection, and $(C)$ injections into the septum label cells that send fibers through the precommissural fornix. The horizontal line passing through the retrogradely labeled cells in field $\mathrm{CA}_{3}$ shows the location of the inset drawings of frontal sections of the hippocampus at the top of the figure. The approximale extent of an injection site in the contralateral hippocampus is shown in the upper right, and the distribution of retrogradely labeled cells is shown in the upper left. The abbreviations used are: DG, dentate gyrus; fi, fimbria; VHC, ventral hippocampal commissure.

dye and for each transport distance, there is every likelihood that the numbers of retrogradely labeled cells in the critical region will not reflect accurately the total population of cells that give rise to collateral projections.

The regio inferior. The large pyramidal cells that comprise field $\mathrm{CA}_{3}$ of Lorente de Nó collectively have the most extensive efferent projections among the hippocampal fields. Autoradiographic studies have established that this field (which forms the greater part of the regio inferior of Cajal) projects to at least six different regions, including the corresponding field of the opposite side, field $\mathrm{CA}_{1}$ on both sides of the brain, the lateral septal nucleus-again on both sides, and the medial part of the subiculum; in addition, there are extensive associational connections within the regio inferior that ascend and descend for various distances along the septotemporal extent of field $\mathrm{CA}_{3}$ (see Swanson and Cowan, 1977; Swanson et al., 1978).

We have reported elsewhere (Swanson et al., 1980) that, after single injections of either true blue, bisbenzimide, or Evan's blue into the septum or into the hippocampus of the same or the opposite side of the brain, essentially all of the pyramidal cells in limited segments of field $\mathrm{CA}_{3}$ can be labeled retrogradely. Conversely, after two or three separate dye injections into the same rcgions, large numbers of doubly or triply labeled cells can be seen. On this basis, we have suggested that all of the known efferents of field $\mathrm{CA}_{3}$ arise as collaterals of the axons of the pyramidal cells.

Now, we have extended these observations and have added two additional findings. First, we currently have experiments (e.g., PS 98, PS 99) in which two different dyes (for example, true blue and bisbenzimide) were injected into the lateral septal nucleus of either side. Such injections result in the double labeling of most of the pyramidal cells in the related parts of fields $\mathrm{CA}_{3}$-on both sides. In some cases (e.g., PS 105, PS 115), the dye injections into the septal nuclei were combined with a third injection (with a different dye, such as Evans blue) into either field $\mathrm{CA}_{1}$ or field $\mathrm{CA}_{3}$ in order to label the association fibers to other levels of $\mathrm{CA}_{3}$ or the Schaffer collateral projections to field $\mathrm{CA}_{1}$ on the same side, as well as the corresponding commissural projections to the opposite side of the hippocampus (Fig. 2). In these brains, more than two-thirds of the pyramidal cells in restricted parts of the regio inferior were triply labeled, thus establishing that the crossed and uncrossed septal, commissural, and associational efferents of field $\mathrm{CA}_{3}$ arise from the same cells.

Second, from experiments with dye injections that have labeled each subfield of the regio inferior selectively (corresponding to Lorente de Nó's subfields $\mathrm{CA}_{3 \mathrm{a}}, \mathrm{CA}_{3 \mathrm{~b}}$, and $\mathrm{CA}_{3 \mathrm{c}}$ ), we now have evidence that each of these regions contributes a commissural projection to the corresponding subfield on the opposite side and that each has a collateral projection (by way of the Schaffer collateral system) to field $\mathrm{CA}_{1}$ on the same side, as well as a crossed projection to the stratum radiatum of field $\mathrm{CA}_{1}$ on the contralateral side. This last conclusion is based on the finding (e.g., experiment PS 217) that injections of true blue that are restricted to different parts of field $\mathrm{CA}_{1}$ on one side consistently label $90 \%$ or more of the pyramidal cells in bilaterally symmetrical zones of field $\mathrm{CA}_{3}$ on the two sides.

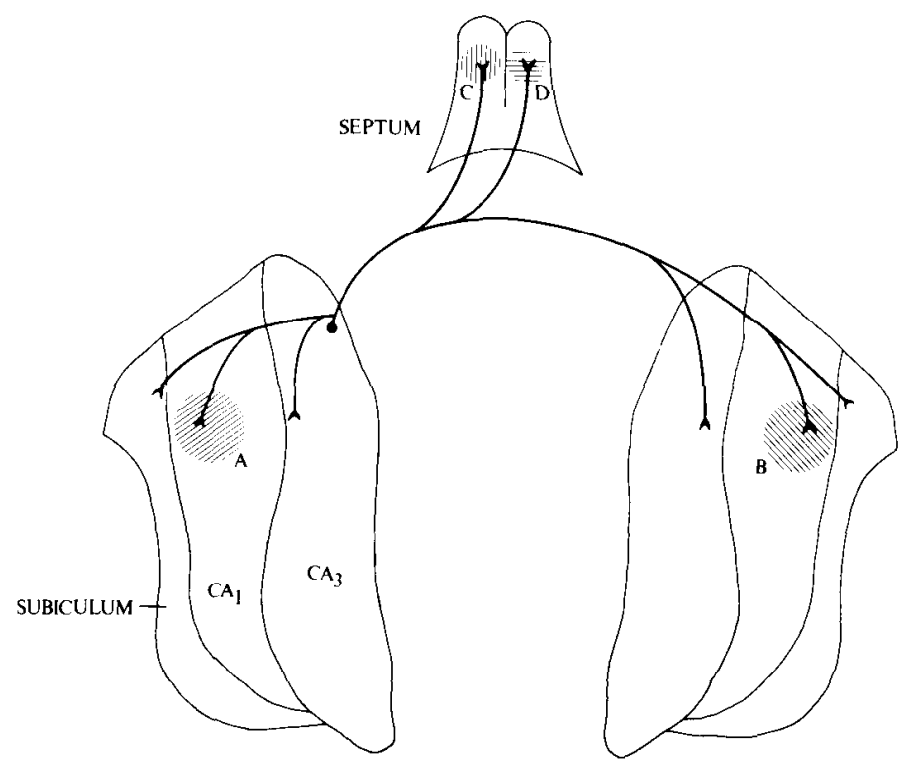

Figure 2. The longer projections that arise in field $\mathrm{CA}_{3}$ are shown superimposed on an unfolded map of Ammon's horn and the subiculum (Swanson et al., 1978). As shown in Table I, various combinations of up to three different dyes were injected into the four terminals fields indicated by the letters $A$ to $D$. 
We have been unable to place localized dye injections within the narrow, medial strip of the subiculum which is known from autoradiographic studies to receive a projection from field $\mathrm{CA}_{3}$. However, after fairly large injections of either true blue or nuclear yellow into the entorhinal area, we have seen occasional retrogradely labeled cells within field $\mathrm{CA}_{3}$. These cells are widely scattered, and even in the best case, no more than about $2 \%$ of the pyramidal cells were labeled. It is difficult to know what the significance of this random labeling of cells in $\mathrm{CA}_{3}$ may be; most probably it reflects the existence of a small number of collaterals that extend into the entorhinal area, beyond the primary retrohippocampal projection of field $\mathrm{CA}_{3}$ to the subiculum, but we cannot rule out the possibility that a small number of neurons in field $\mathrm{CA}_{3}$ project to the entorhinal cortex proper.
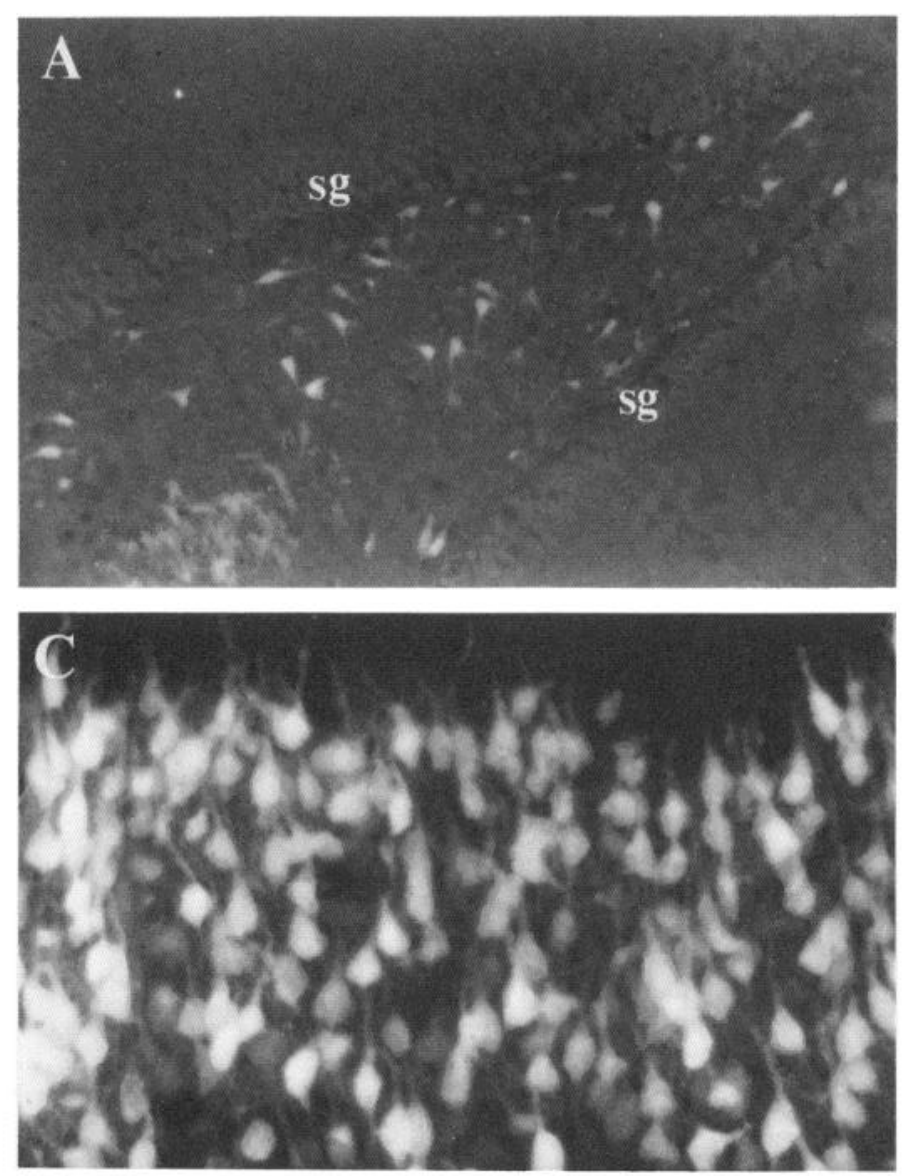

It is worth noting that, after dye injections into either the septum or the hippocampus, the cells in field $\mathrm{CA}_{3}$ of the contralateral side are always labeled less intensely than those in the corresponding regions on the side of the injection (see Fig. 3, $C$ and $D$ ). This difference has been seen after post-injection survival times of up to 6 days, as well as after shorter intervals, so that it is unlikely to be due to the minor differences in transport distance (at least not after bilateral septal injections). However, until more is known about the uptake, transport, and binding properties of these fluorescent dyes, we can only speculate that it might reflect some difference in the size and density of the terminal ramifications of the projections to the ipsilateral and contralateral sides.

The regio superior. Autoradiographic and axonal degeneration studies have established that the pyramidal cells in the regio superior (which is equivalent to Lorente
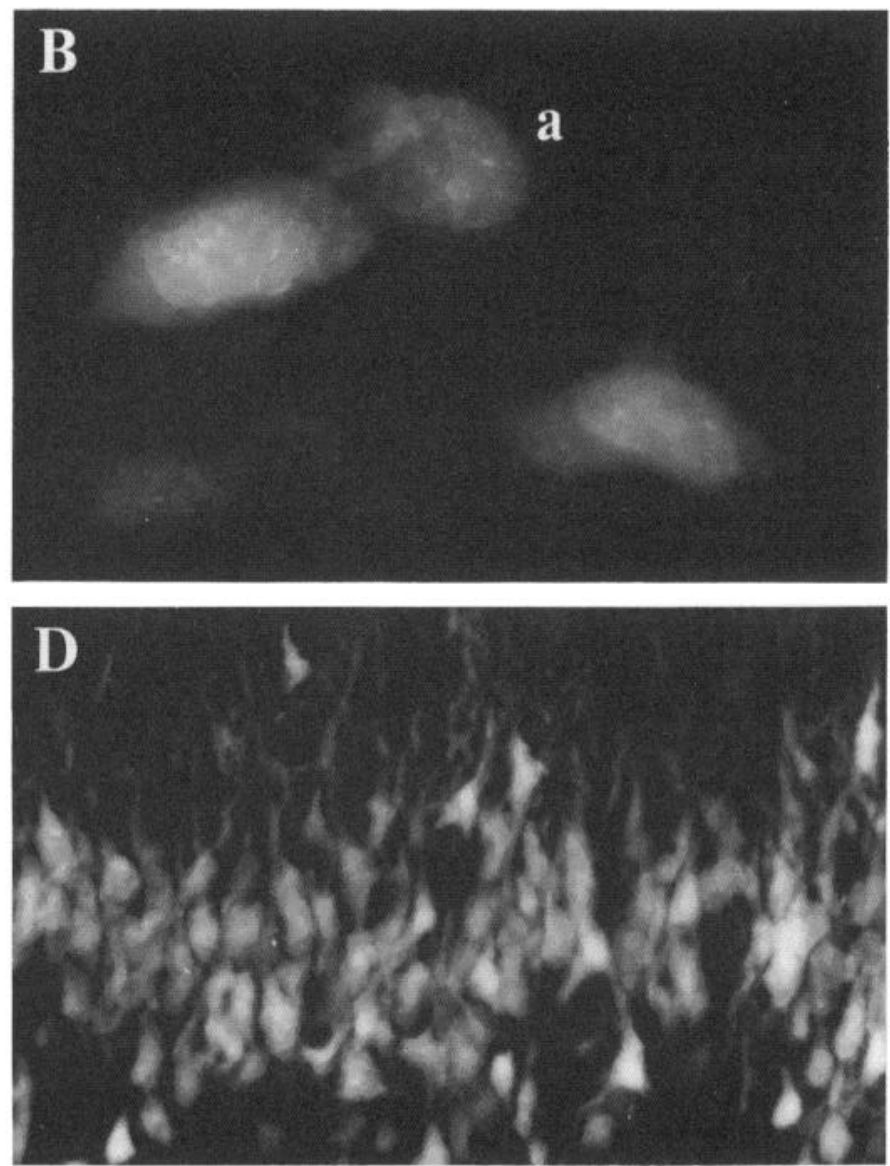

Figure 3. A, A low power photomicrograph to show the distribution of retrogradely labeled cells in the hilar region on the left side of the brain after an injection of true blue at more temporal levels of the dentate gyrus on the same side and an injection of bisbenzimide centered in the dentate gyrus on the contralateral side. The hilar region lies between the two blades of the stratum granulosum $(\mathrm{sg})$ of the dentate gyrus. The crest region of the dentate gyrus is in the upper right of the micrograph; the distal part of subfield $\mathrm{CA}_{3 \mathrm{c}}$ (which also contains retrogradely labeled cells) is in the lower left. Magnification $\times 70$. B, Three doubly labeled neurons in the limiting subzone of the hilar region of the dentate gyrus. In this experiment, which is shown schematically in Figure 5, true blue was injected into the dentate gyrus and adjacent parts of Ammon's horn on the side contralateral to the cells shown in this figure, and bisbenzimide was injected into the corresponding region on the ipsilateral side. When viewed in the fluorescence microscope, the cytoplasm of these cells appears blue (as a result of the accumulation of true blue) and the nucleus appears yellow (because of the binding of bisbenzimide). The cytoplasm in cell $a$ was only faintly fluorescent and faded while this field was being photographed. Magnification $\times 800 . C$ and $D$, The density of retrograde labeling in subfield $C A_{3 b}$ on the same $(C)$ and on the opposite side $(D)$ after a true blue injection into the more temporal parts of field $C_{1}$. Note that the contralateral labeling of individual cells is less intense. The difference in the thickness of the cellular layers on the two sides is due to an asymmetry in the plane of sectioning. 
de Nó's field $\mathrm{CA}_{1}$ ) project rostrally to the lateral septal nucleus and caudally to the subiculum and the entorhinal area on the same side of the brain (Hjorth-Simonsen, 1973; Swanson and Cowan, 1977; Swanson et al., 1978). In addition, there appear to be fairly extensive associational connections from one part of the field to another; these connections are generally rather short and mainly organized along the longitudinal dimension of the field (Lorente de Nó, 1934). To determine whether the major extrinsic projections of $\mathrm{CA}_{1}$ are due to collaterals of the pyramidal cell axons, we have carried out two types of double-labeling experiments (Fig. 4). In the first, true blue was injected into the dorsal part of the lateral septum and bisbenzimide was injected into the dorsal subiculum of the same side. In these cases (e.g., experiment PS 193) and in others in which the dye injections were reversed (e.g., experiment PS 203), more than 90\% of the pyramidal cells in the rostral part of field $\mathrm{CA}_{1}$ were found to be doubly labeled (Fig. $6 B$ ). In the second group of experiments, true blue was injected into the entorhinal cortex of one side, and bisbenzimide was injected into the septum of the same side. In the most successful of these experiments (that is, when the dye injections were optimally placed in both locations), at least $90 \%$ of the pyramidal cells in field $\mathrm{CA}_{1}$ were doubly labeled. It was also apparent in many of these brains that virtually all of the cells could be labeled by an entorhinal injection (of either dye) even when the injected dye did not spread into the subiculum.

As the fibers from $\mathrm{CA}_{1}$ have to pass through the subiculum to reach the entorhinal cortex, it is impossible with this method to label selectively the subicular projection. However, since we can label retrogradely virtually all of the cells by injections into the entorhinal cortex, it follows that the subicular input from field $\mathrm{CA}_{1}$ must arise as a collateral projection from that to the

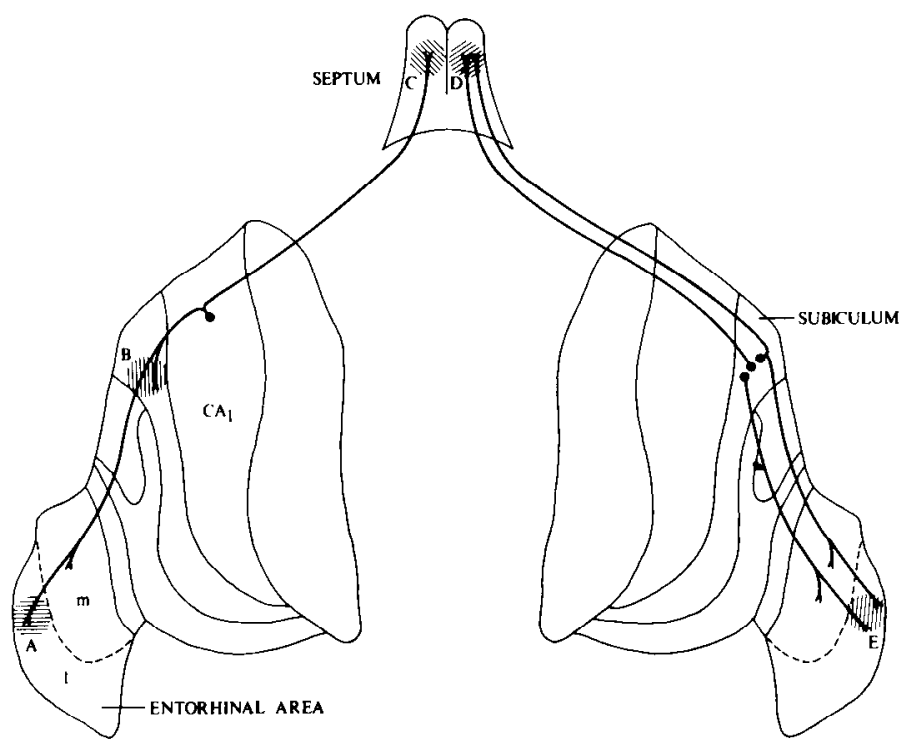

Figure 4. This figure shows the lo ger projections arising from field $\mathrm{CA}_{1}$ (left) and the subiculum (right) and the location of the dye injections ( $A$ to $E$ ) that have been used to label retrogradely the cells of origin of the efferent projections of these two regions. The medial and lateral parts of the entorhinal area are designated $m$ and $l$, respectively. entorhinal cortex. However, it does not follow from this that the axons of all pyramidal cells in field $\mathrm{CA}_{1}$ give off collaterals as they pass through the subiculum. The resolution of that issue lies beyond the capacity of our present techniques.

Although most of the pyramidal cells in field $\mathrm{CA}_{1}$ are aligned within the stratum pyramidale, this layer is not homogeneous throughout the field. In many regions, especially caudally toward its junction with the subicular complex, the cells are distributed in two zones, a more diffuse, deeper zone adjacent to the stratum oriens and a more compact, superficial layer adjacent to the stratum radiatum (Lorente de Nó, 1934). In the rat, there are also significant numbers of apparently displaced pyramidal cells in the stratum radiatum. After dye injections into the septum, the subiculum, or the entorhinal cortex, labeled cells were always found in all three zones (Fig. $6 \mathrm{~A}$ ), which suggests that, at least from the point of view of their efferent projections, the three populations of cells are not dissimilar (see also Chronister and DeFrance, 1979).

Finally, we should mention that, after injections into Ammon's horn which did not spread into the subiculum or the entorhinal area, we have seen occasional labeled neurons in field $\mathrm{CA}_{1}$ of the contralateral side. Although, in any one section, there are seldom more than two or three such cells, this finding is of interest because it is generally believed that field $\mathrm{CA}_{1}$ does not have a commissural projection. It is perhaps a measure of the sensitivity of the dye-labeling technique that such a small number of commissurally projecting neurons can be identified; almost certainly such a minor projection would not have been recognized with the HRP method, and we have not been able to identify it in our autoradiographic material (Gottlieb and Cowan, 1973; Swanson et al., 1978)

The hilar region of the dentate gyrus. From both autoradiographic and HRP-labeled material, it has become clear that the cells of origin of the commissural and ipsilateral associational connections of the dentate gyrus lie within the deep part of the hilus of the area dentata and that earlier axonal degeneration experiments which suggested that they arose from subfield $\mathrm{CA}_{3 \mathrm{c}}$ or field $\mathrm{CA}_{4}$ were mistaken. In an attempt to determine whether these two projection systems arise from separate neuronal populations within the hilar region or as collaterals from the same neurons, we have made injections of true blue into the dentate gyrus of one side, at about the middle of its septotemporal extent, and of bisbenzimide into the corresponding region on the opposite side (Fig. 5). In each successful case (e.g., PS 205 and PS 235), the injected dyes extended for some distance into the molecular layer of the dentate gyrus, as well as into the adjoining hippocampal fields. Since it is known that both the commissural and associational pathways from the mid-portion of the hilus are widely distributed along the septotemporal extent of the inner third of the molecular layer (Gottlieb and Cowan, 1973; Fricke and Cowan, 1978; Swanson et al., 1978), we had assumed that it would be relatively easy to identify either singly or doubly labeled neurons in the deep hilar zone some distance rostral to the injection site. However, in practice, it proved to be 


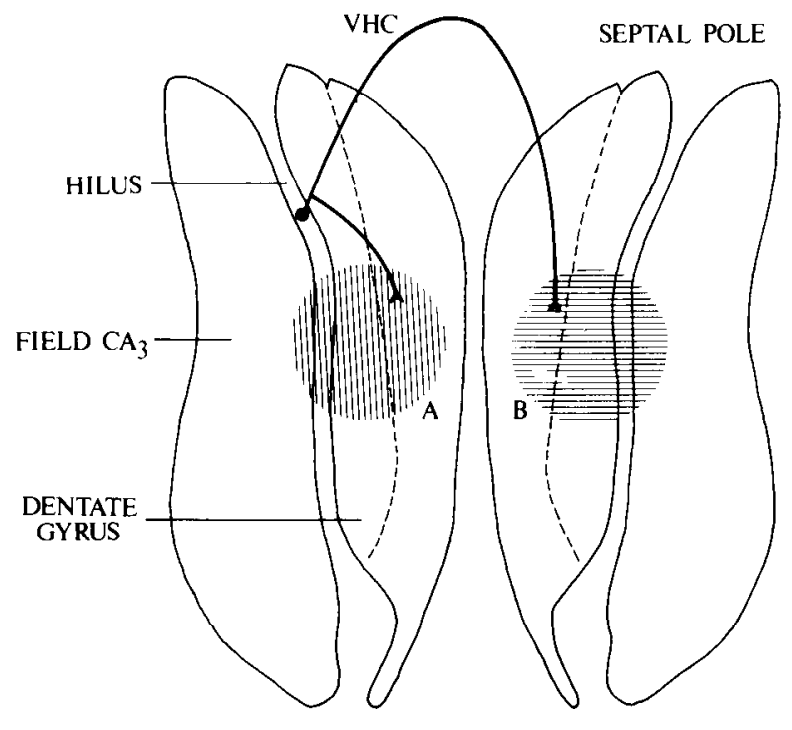

TEMPORAL POLE

Figure 5. A schematic diagram of the longer projections of neurons in the deep hilar region of the dentate gyrus superimposed on an unfolded map of Ammon's horn and the dentate gyrus. Injections centered in the mid-septotemporal portion of the dentate gyrus of one side label neurons in the hilar zone that give rise to descending associational $(A)$ and to commissural $(B)$ projections that terminate within the inner third of the molecular layer (in the so-called commissural-associational zone of the dentate gyrus). The abbreviation used is: VHC, ventral hippocampal commissure.

more difficult to label the cells of origin of these two pathways than any of the others that we have examined in this study. The reasons for this are not clear but are possibly to be found in the diffuse distribution of the fibers in the inner part of the molecular layer. This may be especially true of the commissural fibers to the suprapyramidal blade of the dentate gyrus (Gottlieb and Cowan, 1972; O'Leary et al., 1980), but we have been surprised to find that, in many experiments in which the cells of origin of the supramammillary projection to the dentate gyrus (Wyss et al., 1979) were labeled heavily, the labeling seen in the hilar region, especially on the contralateral side, was not only relatively light but also rather restricted in its septotemporal distribution.

Despite this difficulty, in our best cases (e.g., brain PS 108 ), more than $80 \%$ of the cells in the deep hilar region were doubly labeled by injections of bisbenzimide into the contralateral dentate gyrus and of true blue into the dentate of the same side (Fig. $3 B$ ). Many of the doubly labeled cells were found in the region just deep to the stratum granulosum in the area identified by Cajal (Ramon y Cajal, 1911) as the "limitant subzone of the polymorphic layer," but some labeled cells were seen also throughout the hilar zone. The appearance of the labeled cells was rather variable: most were fusiform or spindle shaped and medium to large in size, with their long dendrites directed more or less parallel to the inner aspect of the stratum granulosum. Others were more generally stellate in shape with no apparent orientation to their dendrites. On the basis of their location and appearance, the labeled cells are probably to be identified with the large "mossy neurons" described in Golgi preparations by Amaral (1978). It is now clear that the majority, if not all, of these cells send their axons into the inner part of the molecular layer of the dentate gyrus of the same side and that they also give rise to collaterals that pass by way of the ventral hippocampal commissure to the corresponding region of the dentate gyrus of the contralateral side.

The subiculum. From autoradiographic and HRP retrograde labeling experiments, it is known that the dorsal part of the subiculum projects to at least four regions: rostrally to the septum, the anterior nuclei of the thalamus, and certain regions of the hypothalamus including the mammillary nuclei, and caudally to the entorhinal cortex (Swanson and Cowan, 1977; Meibach and Siegel, 1977; Swanson et al., 1978). We have examined only two of these projections in the present experiments, namely those to the septum and to the entorhinal cortex, using the same material as was prepared for the analysis of the connections of Ammon's horn (Table I; Fig. 4). To this degree, our observations on the subiculum are less complete than for the other parts of the hippocampal formation, but since the findings suggest that the organization of its efferents may be significantly different from that seen in Ammon's horn, it seems worthwhile placing them on record.

The most striking finding in our experiments with various combinations of injections into the septum and entorhinal cortex is that, even in the zone of maximal labeling in the dorsal part of the subiculum, only about one-third of the cells were doubly labeled. Conversely, in the same zone, many of the cells were clearly labeled either by the dye injected into the entorhinal cortex or by that injected into the septum-but not by both dyes (Fig. 6D). In every case, the singly and doubly labeled neurons appeared to be distributed randomly within the principal cell layer; at least we have not been able to detect any systematic arrangements of the cells that project either to the entorhinal cortex, to the septum, or to both structures.

It seems unlikely that the occurrence of singly labeled neurons is attributable to some inadequacy in the labeling procedure or in the transport of the various dyes because, in the same brains, the pyramidal cells in the immediately adjoining part of field $\mathrm{CA}_{1}$ showed extensive double labeling. Rather, it would appear that the organization of the efferents from the dorsal part of the subiculum is basically different from that of the fields of Ammon's horn, in that some of its projection neurons send their axons exclusively to the entorhinal cortex, while others only project rostrally; only a minority appears to have collateral projections to the septum and the entorhinal cortex. We cannot, at present, rule out the possibility that the neurons that were singly labeled in our experiments did not have collateral projections to other regions. If such collaterals exist, it seems unlikely that they will be found to project to the diencephalon because almost certainly, they would have been labeled by our dye injections as they pass through the septum. Finally, it is worth noting that the ventral subiculum may be organized somewhat differently again, since it was possible to label virtually all of the cells in this region after septal injections of true blue or bisbenzimide (Fig. 

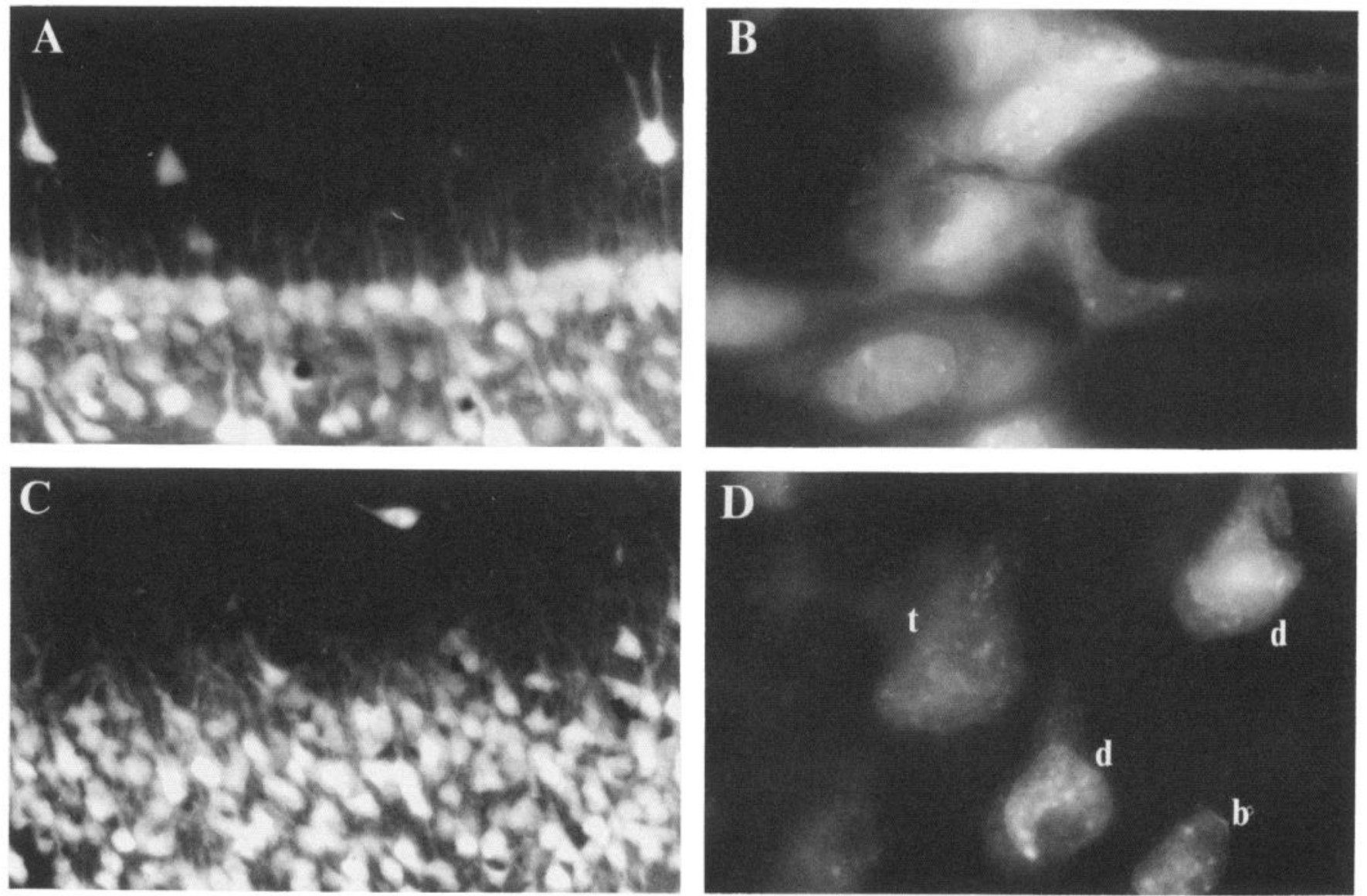

Figure 6. A, Retrograde labeling in subfield $\mathrm{CA}_{1}$ after an injection of true blue in the septal region on the same side of the brain. Note the dense labeling of both deep and superficial pyramidal cells and also of the scattered cells in the stratum radiatum. Magnification $\times 175 . B$, Doubly labeled neurons in the superficial row of pyramidal cells in subfield $\mathrm{CA}_{\mathrm{lc}}$ after an injection of true blue in the lateral entorhinal area and an injection of bisbenzimide in the septal region (see injection sites $A$ and $C$ on the left side of Fig. 4). The labeled apical dendrites of these pyramidal cells here are oriented horizontally and to the right. Magnification $\times$ 800. $C$, Retrogradely labeled cells in the ventral part of the subiculum after an injection of true blue in the septal region. Note the labeled cell in the molecular layer (upper half of figure). Magnification $\times 175 . D$, Retrograde labeling in the dorsal part of the subiculum after a true blue injection in the entorhinal area (at site $E$ in Fig. 4) and a bisbenzimide injection in the septal region (site $D$ in Fig. 4). Retrograde labeling in the dorsal part of the subiculum is never as dense as in the ventral part after septal or entorhinal injections. This photomicrograph was taken through the zone of maximal double labeling and shows two doubly labeled cells $(d)$, a cell labeled only by the bisbenzimide injection (nucleus indicated at $b$ ), and a cell labeled only with true blue (cytoplasm indicated at $t$ ). Magnification $\times 800$.

$6 C)$. This is of interest because the diencephalic projection of the ventral subiculum is known to be different from that of the dorsal subiculum (in particular, it projects to the ventromedial nucleus of the hypothalamus rather than to the mammillary complex (Swanson and Cowan, 1977)).

\section{Discussion}

Three general conclusions may be drawn from the findings in this study. (i) Each of the efferent projections of the regio inferior and the regio superior of Ammon's horn arises as a collateral from the axons of pyramidal cells in the relevant field (or subfield). (ii) The associational and commissural projections to the molecular layer of the dentate gyrus similarly arise as collaterals of the axons of the medium to large fusiform and stellate cells in the deep hilar region of the area dentata. (iii) The organization of the projection neurons in the dorsal part of the subiculum is basically different from that of either
Ammon's horn or the neocortex in that it consists of neurons that project either to the entorhinal cortex, to the septum, or to both areas, and the cells with the different efferent projections appear to be intermingled randomly and are not segregated into distinct laminae or sublaminae. Before discussing some of the implications that arise from these conclusions, it is important to consider the validity of the evidence on which they are based and to examine the advantages and limitations of the dye-labeling procedures that we have used.

Methodological considerations. The fluorescent dyes that we have used offer several advantages over the more widely used HRP method for the retrograde labeling of neurons. First, as we have seen in the present experiments and as has been found in other systems where it has been possible to provide quantitative estimates of the numbers of neurons labeled from different regions (for example, the paraventricular nucleus (Sawchenko and Swanson, 1981)), the fluorescent dyes appear to be 
appreciably more sensitive than HRP, and for this reason, their use has brought to light pathways that have been difficult to determine by other means. We have been especially impressed that it has been possible to label essentially all of the pyramidal cells in both the regio superior and the regio inferior after appropriately placed injections of all four of the dyes that we have used, whereas with HRP, we were never able to label more than about $30 \%$ to $40 \%$ of the cells, even in the zones of maximum labeling. The reasons for the apparently greater sensitivity of the fluorescent dyes are not known; conceivably, it could be due to one or more of several factors, including the greater affinity of the axon terminals for the dyes, their enhanced uptake for retrograde transport, the nature of their accumulation in the neuronal perikarya (including the likelihood that they are not degraded rapidly in vivo), and their ready visualization in the fluorescence microscope.

A sccond, obvious advantage of the dyes is that they permit the direct demonstration of collateral projections. This is especially true when true blue and bisbenzimide are used, for not only do they label different cellular components (the perikaryon and nucleus, respectively) but their emission spectra are also quite different so that they can be viewed and photographed with the same filter system. Both of these dyes have proved equally useful for all of the axonal projection systems that we have examined, and in this respect, they are decidedly superior to Evans blue, which has been less valuable for demonstrating the longer projections, such as those to the septum and to the hippocampus of the opposite side. Whether this is due only to the length of the relevant axons (and, in consequence, the distance over which the dye has to be transported retrogradely) or to some other factor is not known; but, empirically, we have found it advantageous in our triple-labeling experiments to limit the use of Evans blue to the shortest projections (usually the ipsilateral association pathways).

In view of these clear advantages, it is important to emphasize that we have not always seen doubly or triply labeled neurons even when the dyes were injected into two or three of the known projections of the hippocampal formation. To demonstrate collateral projections unequivocally, it is important that the dyes be injected not only into the correct field(s) but also into the correct part(s) of each field. Since we know from autoradiographic studies that the projections of each field are topographically arranged, it follows that, for any given sector of a pyramidal cell field to be adequately labeled, the dye must be injected either into (or at least very close to) the topographically related part of the projection field. And where two or more dyes are used, each must be injected into the appropriate region within the overall terminal field if the collaterals are to be labeled optimally. This point is well illustrated by some of our experiments on field $\mathrm{CA}_{1}$, in which true blue was injected into the entorhinal cortex and bisbenzimide was injected into the septum. In one case (experiment PS 214), essentially all of the pyramidal cells in the relevant part of $\mathrm{CA}_{1}$ were doubly labeled; but in a second experiment (PS 194), the doubly labeled cells were confined to a fairly narrow rostrocaudal band (about $400 \mu \mathrm{m}$ wide), and surrounding this zone, there was an extensive area in which the cells were labeled with either true blue or bisbenzimide. Had the findings in the first experiment not been so clear cut, it would have been difficult to interpret those in the second case, especially since the topographic arrangement of the projection of $\mathrm{CA}_{1}$ upon the entorhinal cortex has not been worked out yet.

In our experience, the true blue-bisbenzimide combination generally has proved to be the most useful in large part because their effective "injection-to-labeling ranges" are similar. As we have pointed out, this does not appear to be true for Evans blue nor, in our experience, is it true for nuclear yellow. This is unfortunate because nuclear yellow generally labels nuclei more brilliantly than bisbenzimide; but, when equal volumes and equal concentrations of these two dyes were injected into the septal region (in different experiments), the zone containing retrogradely labeled neuronal nuclei was invariably smaller after the nuclear yellow injections, even though the extent of the injections seemed more or less equivalent.

Another methodological point that should be made concerns the tendency of the retrogradely transported dyes to escape from the labeled neurons and to label surrounding cells secondarily. This is especially troublesome after relatively long post-injection times and when bisbenzimide and nuclear yellow are used (Bentivoglio et al., 1980a; Swanson and Kuypers, 1980). Although most of the dye that escapes seems to be taken up preferentially by the surrounding glial cells, where one is dealing with a densely packed population of neurons, as in the stratum pyramidale of the hippocampus, the possibility that neighboring pyramidal cells may be labeled "indirectly" poses a serious problem, and all the more so in view of the recent report of electrical coupling between cells in field $\mathrm{CA}_{3}$ (MacVicar and Dudek, 1980). To minimize this possibility, we have tried to keep our postinjection survival times as short as possible and have confirmed every important finding with true blue and Evans blue which seem to escape much less readily. For these reasons, we feel fairly confident that our observations of multiple collateral projections are valid, and in support of this position, we may cite the fact that, in nearly every experiment, we could define the boundaries of the retrogradely labeled zone precisely. Over the space of just $20 \mu \mathrm{m}$, one often passed from a zone containing intensely labeled cells to one in which none of the cells contained the dye. A striking example of this is at the boundary between $\mathrm{CA}_{1}$ and the subiculum after labeling the Schaffer collateral system, and as we have pointed out, within the subiculum itself, labeled and unlabeled neurons often lay side by side or one neuron might be labeled with bisbenzimide and an adjacent neuron with true blue, without the slightest indication that the dyes had spread from one cell to the other. In this context, we should emphasize that our findings in field $\mathrm{CA}_{3}$ are essentially no different than those for field $\mathrm{CA}_{1}$ in which the cells do not appear to be electrically coupled (Knowles and Schwartzkroin, 1981).

Hippocampal projections. Although we have not attempted to label every known projection field of the hippocampus or subiculum, our observations are suffi- 
ciently consistent to enable us to conclude that, from the point of view of their efferent projections, the pyramidal cells in the regio inferior and the regio superior of Ammon's horn are fundamentally homogeneous populations. It remains to be seen whether every pyramidal cell in each region has collateral projections to all of the known projection areas of the field as a whole. On the grounds that, after essentially every injection that we have made, virtually all of the pyramidal cells in some part of field $\mathrm{CA}_{1}$ or $\mathrm{CA}_{3}$ could be labeled, this seems likely; however, it remains possible that a small percentage of the cells in both fields lacks one or more of the typical collaterals.

In this respect, the dorsal part of the subiculum is quite different from the fields of Ammon's horn, for here, only a minority of the cells could be doubly labeled by injections into the entorhinal cortex and the septum. In the light of our findings in Ammon's horn, it seems unlikely that this observation is due to some technical limitation in the dye-labeling procedure or to incorrect placement of the injections. Instead, it would seem to point to a true heterogeneity in the projecting cells of the subiculum, such that some neurons project to the entorhinal cortex, others to the septum, and some (amounting to about $30 \%$ ) project to both regions. What is especially intriguing is that these three groups of cells seem to be intermingled randomly and are not segregated into different layers as are the cells in the neocortex which project to other cortical fields, to the thalamus, and to various other subcortical structures (Gilbert and Kelly, 1975; Jones and Wise, 1977; Wise et al., 1979; Kawamura and Chiba, 1979). It would be interesting to know whether the cells of origin of the other efferents of the subiculum are intermingled similarly or if they are segregated spatially from those that project to the septum and entorhinal cortex.

Our results have also gone a long way toward clarifying the origin of the associational and commissural afferents to the dentate gyrus. These were originally thought to arise from cells in fields $\mathrm{CA}_{3}$ and $\mathrm{CA}_{4}$ of Lorente de Nó (Zimmer, 1971; Gottlieb and Cowan, 1973), but later experimental studies, involving the retrograde transport of HRP, have focused attention on the fusiform cells in the deep part of the hilar region as the probable source of the commissural afferents, and by inference, also of the associational fibers (Swanson et al., 1978; West et al., 1979). The present findings have confirmed that these cells do indeed project to the contralateral dentate gyrus and also have established that their axons give off collaterals to the dentate gyrus of the ipsilateral side. Although we have not been able to demonstrate that all of the cells in this part of the hilar region can be doubly labeled by injecting different dyes into the molecular layer of the dentate gyrus on the two sides, the fact that more than $80 \%$ can be so labeled suggests that this is likely. Of course, this does not mean that the two collateral systems have identical projections; other evidence, mainly from autoradiographic experiments, indicates that the ipsilateral associational projections are generally more extensive (in the longitudinal dimension of the dentate gyrus) and that they tend to dominate the suprapyramidal blade of the gyrus (Gottlieb and Cowan, 1973; Fricke and
Cowan, 1978; O'Leary et al., 1979). However, as we shall discuss below, the fact that the two projections arise as collaterals from the same neurons has important implications both for their development and for their response to selective deafferentation.

Implications for development and plasticity in the hippocampal formation. From autoradiographic experiments in which the commissural and associational fibers to the dentate gyrus were labeled by injections of ${ }^{3} \mathrm{H}$ amino-acids and their relative distributions on the two sides were determined by systematic grain counts, Gottlicb and Cowan (1972) suggested that, during their development, these two fiber systems compete with each other for the available synaptic sites within the inner part of the molecular layer and that the outcome of this competition is determined in part by the time at which the granule cells in the supra- and infrapyramidal blades are generated and in part by the time of arrival of the two groups of afferents. Since both pathways terminate upon the dendrites of the granule cells within a narrow zone (occupying approximately the inner one-third of the molecular layer), they suggested that the fibers might share the same cytochemical specificity. In the intervening years, several different lines of evidence have been adduced in support of what has come to be known as the "temporal hypothesis" (Fricke and Cowan, 1978; O'Leary et al., 1979), but until our finding that the two groups of fibers arise from the same cells in the hilar region, there has been no direct evidence to support the view that they share a common specificity.

One of the few generalizations that can be made regarding the connections of the hippocampus is that each of its commissural systems (including those to the dentate gyrus, to field $\mathrm{CA}_{1}$, and to the various subfields of the regio inferior) is matched by a parallel system of associational connections on the ipsilateral side and that the parallelism extends not only to the topographic regions and laminar zones in which the two systems terminate but also to the intrahippocampal course that their fibers follow (Gottlieb and Cowan, 1973; Swanson et al., 1978). The present finding that all of these systems arise as collateral projections suggests that the "rules" governing their development may be simpler than had been expected; this certainly would be the case if the associational and crossed projections share the same cytochemical specificities.

The finding that the commissural and associational afferents to the dentate gyrus arise from the same neurons also bears significantly on the interpretation of some recent experiments on plasticity in this system. O'Leary et al. (1979) have found that, when the commissural fibers to the dentate gyrus are interrupted (in young adult rats), the associational fibers show a prompt sprouting response, and within a few weeks, they appear to occupy all of the vacated synaptic sites in the inner third of the molecular layer (see also McWilliams and Lynch, 1978). At the time, this response was viewed simply as a form of "reactive synaptogenesis" (Cotman and Nadler, 1978) induced by the degeneration of a neighboring fiber system. It is now evident that the response is more complicated than this. Sectioning the hippocampal commissure not only interrupts the commissural fibers to the 
contralateral dentate gyrus but also partially axotomizes the cells of origin of the associational system. That is to say, the response of the associational system has to be viewed both as a sprouting reaction and as a response to axonal injury. In this respect, it may be thought of as a "pruning response" of the type first described by Schneider (1973). If this type of pruning response is secn as the attempt by partially axotomized neurons to maintain an optimal number of synaptic connections in the dentate gyrus, one would expect to see an appreciable expansion of the associational systems after commissurotomy. This, in fact, is what has been observed; indeed, after commissural lesions, the associational fibers to the infrapyramidal blade of the dentate gyrus form about twice as many synapses as they do in normal animals (O'Leary et al., 1980). As yet, no study of the response of the other hippocampal associational systems to commissurotomy has been reported, but in light of the present findings, it seems reasonable to predict that they too will show similar pruning and sprouting reactions.

\section{References}

Amaral, D. G. (1978) A Golgi study of cell types in the hilar region of the hippocampus in the rat. J. Comp. Neurol. 182: 851-914.

Bentivoglio, M., H. G. J. M. Kuypers, C. E. Catsman-Berrevoets, and O. Dann (1979) Fluorescent retrograde neuronal labeling in rat by means of substances binding specifically to adenine-thymine-rich DNA. Neurosci. Lett. 12: 235-240.

Bentivoglio, M., H. G. J. M. Kuypers, and C. E. CatsmanBerrevoets (1980a) Retrograde neuronal labeling by means of bisbenzimide and nuclear yellow (Hoechst S 769121). Measures to control diffusion of the tracers out of retrogradely labeled neurons. Neurosci. Lett. 18: 19-24.

Bentivoglio, M., H. G. J. M. Kuypers, C. E. Catsman-Berrevoets, H. Loewe, and O. Dann (1980b) Two new fluorescent retrograde neuronal tracers which are transported over long distances. Neurosci. Lett. 18: 25-30.

Chronister, R. B., and J. F. DeFrance (1979) Organization of projection neurons of the hippocampus. Exp. Neurol. 66: 509523.

Chronister, R. B., R. W. Sikes, and L. E. White, Jr. (1975) Postcommissural fornix: Origin and distribution in the rodent. Neurosci. Lett. 1: 199-201.

Cotman, C. W., and J. V. Nadler (1978) Reactive synaptogenesis in the hippocampus. In Neuronal Plasticity, C. W. Cotman, ed., pp. 227-271, Raven Press, New York.

De Olmos, J., and L. Heimer (1977) Mapping of collateral projections with the HRP-method. Neurosci. Lett. 6: 107114.

Fricke, R., and W. M. Cowan (1978) An autoradiographic study of the commissural and ipsilateral hippocampo-dentate projections in the adult rat. J. Comp. Neurol. 181: 253-270.

Geisert, E. E. (1976) The use of tritiated horseradish peroxidase for defining neuronal pathways: A new application. Brain Res. 117: 130-135.

Gilbert, C. D., and J. P. Kelly (1975) The projections of cells in different layers of the cat's visual cortex. J. Comp. Neurol. 163: 81-105.

Gottlieb, D. I., and W. M. Cowan (1972) Evidence for a temporal factor in the occupation of available synaptic sites during the development of the dentate gyrus. Brain Res. 41: 452-456.

Gottlieb, D. I., and W. M. Cowan (1973) Autoradiographic studies of the commissural and ipsilateral association connections of the hippocampus and dentate gyrus of the rat. I. The commissural connections. J. Comp. Neurol. 149: 393-422.
Hjorth-Simonsen, A. (1973) Some intrinsic connections of the hippocampus in the rat: An experimental analysis. J. Comp. Neurol. 147: 145-162.

Hjorth-Simonsen, A., and B. Laurberg (1977) Commissural connections of the dentate area in the rat. J. Comp. Neurol. 174: 591-606.

Jones, E. G., and B. K. Hartman (1978) Recent advances in neuroanatomical methodology. Annu. Rev, Neurosci. 1: 215296.

Jones, E. G., and R. Y. Leavitt (1974) Retrograde axonal transport and the demonstration of non-specific projections to the cerebral cortex and striatum from thalamic intralaminar nuclei in the rat, cat and monkey. J. Comp. Neurol. 154: 349378.

Jones, E. G., and S. P. Wise (1977) Size, laminar and columnar distribution of efferent cells in the sensory-motor cortex of monkeys. J. Comp. Neurol. 175: 391-438.

Kawamura, K., and M. Chiba (1979) Cortical neurons projecting to the pontine nuclei in the cat. An experimental study with the horseradish peroxidase technique. Exp. Brain Res. 35: 269-285.

Knowles, W. D., and P. A. Schwartzkroin (1981) Local circuit synaptic interactions in hippocampal brain slices. J. Neurosci. 1: 318-322.

Kuypers, H. G. J. M., C. E. Catsman-Berrevoets, and R. E. Padt (1977) Retrograde axonal transport of fluorescent substances in the rat's forebrain. Neurosci. Lett. 6: 127-135.

Kuypers, H. G. J. M., M. Bentivoglio, D. Van der Kooy, and C. E. Catsman-Berrevoets (1979) Retrograde transport of bisbenzimide and propridium iodide through axons to their parent cell bodies. Neurosci. Lett. 12: 1-7.

LaVail, J. H., and M. M. LaVail (1972) Retrograde axonal transport in the central nervous system. Science 176: 14161417.

Lorente de Nó, R. (1934) Studies on the structure of the cerebral cortex. II. Continuation of the study of the Ammonic system. J. Psychol. Neurol. (Lpz.) 46: 113-177.

MacVicar, B. A., and F. E. Dudek (1980) Dye-coupling between $\mathrm{CA}_{3}$ pyramidal cells in slices of rat hippocampus. Brain Res. 196: 494-497.

McWilliams, R., and G. Lynch (1978) Terminal proliferation and synaptogenesis following partial deafferentation. The reinnervation of the inner molecular layer of the dentate gyrus following removal of its commissural afferents. J. Comp. Neurol. 180: 581-616.

Meibach, R. L., and A. Siegel (1975) The origin of fornix fibers which project to the mammillary bodies in the rat: $A$ horseradish peroxidase study. Brain Res. 88: 508-512.

Meibach, R. C., and A. Siegel (1977) Efferent connections of the hippocampal formation in the rat. Brain Res. 124: 197224.

Mesulam, M. -M. (1976) The blue reaction product in horseradish peroxidase histochemistry. Incubation parameters and visibility. J. Histochem. Cytochem. 24: 1273-1280.

O'Leary, D. D. M., R. A. Fricke, B. B. Stanfield, and W. M. Cowan (1979) Changes in the associational afferents to the dentate gyrus in the absence of its commissural inputs. Anat. Embryol. (Berl.) 156: 283-299.

O'Leary, D. D. M., B. B. Stanfield, and W. M. Cowan (1980) Evidence for the sprouting of the associational fibers to the dentate gyrus following removal of the commissural afferents in adult rats. Anat. Embryol. (Berl.) 159: 151-161.

Ramón y Cajal, S. (1911) Histologie du système nerveux de l'homme et des vertébrés, Vol. 2, S. A. Maloine, Paris.

Sawchenko, P. E., and L. W. Swanson (1981) A method for tracing biochemically defined pathways in the central nervous system using combined fluorescence retrograde transport and immunohistochemical techniques. Brain Res., 210: $31-$ 51 . 
Schneider, G. E. (1973) Early lesions of superior colliculus: Factors affecting the formation of abnormal retinal projections. Brain Behav. Evol. 8: 73-109.

Steward, O., S. A. Scoville, and S. L. Vinsant (1977) Analysis of collateral projections with a double retrograde labeling technique. Neurosci. Lett. 5: 1-5.

Swanson, L. W., and W. M. Cowan (1975) Hippocampo-hypothalamic connections: Origin in subicular cortex, not Ammon's horn. Science 189: 303-304.

Swanson, L. W., and W. M. Cowan (1977) An autoradiographic study of the organization of the efferent connections of the hippocampal formation in the rat. J. Comp. Neurol. 172: 4984.

Swanson, L. W., and H. G. J. M. Kuypers (1980) A direct projection from the ventromedial nucleus and retrochiasmatic area of the hypothalamus to the medulla and spinal cord of the rat. Neurosci. Lett. 17: 307-312.

Swanson, L. W., J. M. Wyss, and W. M. Cowan (1978) An autoradiographic study of the organization of intrahippocampal association pathways in the rat. J. Comp. Neurol. 181: 681-716.

Swanson, L. W., P. E. Sawchenko, and W. M. Cowan (1980)
Evidence that the commissural, associational and septal projections of the regio inferior of the hippocampus arise from the same neurons. Brain Res. 197: 207-212.

Van der Kooy, D., H. G. J. M. Kuypers, and C. E. CatsmanBerrevoets (1978) Single mammillary cells with divergent axon collaterals. Demonstration by a simple, fluorescent retrograde double-labeling technique in the rat. Brain Res. 158: 189-196.

West, J. R., H. O. Nornes, C. L. Barnes, and M. Bronfenbrenner (1979) The cells of origin of the commissural afferents to the area dentata in the mouse. Brain Res. 160: 203-216.

Wise, S. P., E. A. Murray, and J. D. Coulter (1979) Somatotopic organization of the corticospinal and corticotrigeminal neurons in the rat. Neuroscience 4: 65-78.

Wyss, J. M., L. W. Swanson, and W. M. Cowan (1979) Evidence for an input to the molecular layer and the stratum granulosum of the dentate gyrus from the supramammillary region of the hypothalamus. Anat. Embryol. (Berl.) 156: 165-176.

Zimmer, J. (1971) Ipsilateral afferents to the commissural zone of the fascia dentata, demonstrated in decommissurated rats by silver impregnation. J. Comp. Neurol. 142: 393-416. 